\title{
OCASO DE LA MEDICINA SOCIAL EN ESPAÑA: EL CASO DE LA LEPTOSPIROSIS
}

\author{
Esteban Rodríguez Ocaña \\ Universidad de Granada \\ erodrig@ugres \\ ORCID iD: http://orcid.org/0000-0003-4195-4487
}

Recibido: 21 diciembre 2016; Aceptado: 22 abril 2017.

Cómo citar este artículo/Citation: Rodríguez Ocaña, Esteban (2017), "Ocaso de la Medicina Social en España: el caso de la leptospirosis", Asclepio 69 (2): p199. doi: http://dx.doi.org/10.3989/asclepio.2017.22

RESUMEN: Con el presente trabajo pretendo explorar el agotamiento de la Sanidad franquista en torno a un ejemplo concreto, el de la lucha contra la leptospirosis en el mundo rural, en especial en torno al cultivo del arroz, comprobando las semejanzas y diferencias de dicho empeño con el de otros anteriores surgidos de la matriz médico-social. Dicha enfermedad endemoepidémica rural fue singularizada como enemigo en la posguerra, por sus consecuencias sobre la producción en momentos delicados del cultivo de arroz, como la siega, si bien no fue hasta 1953 cuando se comenzó a actuar con algún sistema, merced al primer acuerdo de asistencia técnica firmado con la OMS, que permitió la venida de Brenno Babudieri, un microbiólogo italiano de fama mundial en este campo, quien probó en España una vacuna. Pero las iniciativas fueron provinciales y no coordinadas, dependientes aparentemente de la voluntad de los Jefes Provinciales de Sanidad y centradas sólo en medidas de laboratorio.

PALABRAS CLAVE: Medicina Social; Franquismo; Leptospirosis; Vacuna; Serología.

\section{LEPTOSPIROSIS AS A CASE STUDY OF THE DECLINE OF SOCIAL MEDICINE IN SPAIN}

ABSTRACT: This paper focuses on a particular case of the public health administration under Franco which shows its decay in the rural areas. Leptospirosis was fought specially in the rice fields, and I afford to evaluate its similarities and disimilarities to previous health campaigns brought about by the idea of Social Medicine. It became a public health hazard after the Civil War, due to its consequences on delicate moments of rice production such as the harvest, although no organised measures were taken until 1953. It was thanks to the first agreement on technical assistance signed with the WHO, that allowed the arrival of Brenno Babudieri, a world famous Italian microbiologist on the matter, who tested a vaccine in Spain. But public health initiatives regarding leptospirosis were laboratory centered, scattered and apparently dependent on the will of the provincial medical officer.

KEY WORDS: Social Medicine; Francoism; Leptospirosis; Vaccine; Serology.

Copyright: (C) 2017 CSIC. Este es un artículo de acceso abierto distribuido bajo los términos de la licencia Creative Commons Attribution (CC BY) España 3.0. 


\section{INTRODUCCIÓN}

La Medicina Social, que parte de una consideración económico-social de la enfermedad fusionada con planteamientos eugenésicos, incorporó a las preocupaciones ambientales de la Higiene decimonónica la captación activa y precoz de los pacientes, una notoria dimensión preceptiva o didáctica y un nuevo espacio asistencial, el centro de salud. Su plasmación mas precoz fueron las distintas luchas sanitarias, verticales, de carácter marcadamente urbano: contra la tuberculosis, las enfermedades venéreas o la mortalidad infantil, de origen y base filantrópicas e intervención municipal como mas caracterizada participación gubernativa (Rodríguez-Ocaña y Molero, 1993; Martínez Antonio y Molero, 2002). En España se les sumó, mas adelante, la lucha contra endemoepidemias rurales tales como el paludismo (Rodríguez-Ocaña et al., 2003), la anquilostomiasis (Rodríguez-Ocaña y Menéndez-Navarro, 2006 y 2009) o el tracoma -esta, mejor caracterizada como enfermedad de la pobreza (Bernabeu-Mestre y Galiana- Sánchez, 2012; Bernabeu-Mestre, et al., 2013; Galiana-Sánchez, et al., 2010). Como tengo advertido en trabajos anteriores, la Salud Pública nació y se desarrolló en el entorno urbano y su extensión al mundo campesino tuvo que vencer importantes obstáculos sociales y prejuicios sanitarios (RodríguezOcaña y Martínez Navarro, 2008; Rodríguez-Ocaña, 2012). En España, en la senda abierta por la intervención antipalúdica y las doctrinas de la Organización de Higiene de la Sociedad de Naciones, tuvo un momento decisivo en la adopción por la Segunda República del modelo jerárquico y territorial de los Centros de Higiene de influencia norteamericana. Los centros secundarios tuvieron la consideración estratégica de "jalones principales de la futura organización sanitaria", entre otras razones porque en ellos se debían concentrar las distintas luchas preexistentes ${ }^{1}$, configurando un modelo de acercamiento integral a la salud de las poblaciones, lo que resultó ser un ideal inalcanzable. El plan original planteaba establecer entre 180 y 200 en ocho años. En diciembre de 1932 se habían creado 16 Centros secundarios, que eran 46 en febrero de 1936 (Rodríguez-Ocaña, 2008). A finales de 1950 existían 77 en actividad, 6 pendientes de inaugurar y 9 en curso de construcción ${ }^{2}$. Como indica esta cifra, la ley de Bases de la Sanidad Nacional de 1944 había mantenido la opción estructural de la República, aunque, paradójicamente, había dotado de personalidad propia a la Higiene infantil y devuelto su entidad particular al Patronato Nacional Antituberculoso, lo que iba en contra de la voluntad expresa de unidad estatalizadora tanto de la República como del Preámbulo y Título Preliminar de la Ley de 1944. Sin embargo, la preferencia franquista por el Seguro Obligatorio de Enfermedad, gestionado por los hombres de Girón de Velasco, restó fuerza a la opción preventivista que ni supo superar los problemas heredados ni los nuevos de la coexistencia con el Seguro (Marset Campos, et al., 1998).

Las distintas luchas fueron comunes a todo el mundo industrial, en cada país con sus componentes particulares, pero dotadas de bases comunes que se generaron y difundieron en red, contando con la participación señalada de instituciones supranacionales efímeras o permanentes, privadas y públicas: Congresos internacionales ad hoc, la Oficina de Higiene de la Sociedad de Naciones, la Cruz Roja, organizaciones particulares como Save The Children entre otras muchas y, de manera prominente, con la actuación ejecutiva de poderosas y riquísimas obras filantrópicas como las norteamericanas Fundación Rockefeller y Fundación Milbank. Tras la II GM la Organización Mundial de la Salud se convirtió en el campo de relación intergubernamental más significativo durante decenios.

Con el presente trabajo pretendo explorar el agotamiento de la Sanidad franquista con un ejemplo concreto, el de la lucha contra la leptospirosis en el mundo rural, en especial en torno al cultivo del arroz, comprobando las semejanzas y diferencias de dicho empeño con el de otros anteriores surgidos de la matriz médico-social. Comenzaré por explicar el descubrimiento de dicha afección y sus causas, su presencia en España y la vinculación de la medicina y sanidad españolas con las redes internacionales de difusión y gestión del conocimiento, para acabar analizando los pormenores de la lucha antileptospirósica y sus peculiaridades.

\section{LEPTOSPIROSIS EN ESPAÑA}

Sobre la leptospirosis, recordemos que se trata de una infección causada por espiroquetas, transmitidas por diversos animales, que produce fiebre, dolores musculares, síntomas meníngeos, hepáticos y renales y que en muchos casos remite espontáneamente, obligando empero a una larga convalecencia.

Del cajón de sastre de las llamadas fiebres biliosas a finales del siglo XIX la agudeza clínica de la escuela de Louis Landouzy (1845-1917), por medio del maestro (Landouzy, 1883) y de Albert Mathieu (1855-1917) (Mathieu, 1886), había singularizado el "tifus hepático" o "ictericia infecciosa", entidad mejor descrita por Adolf Weil (1848-1916) (Weil, 1886), en lo que pasó a ser conocido como enfermedad de Weil o fiebre icte- 
rohemorrágica. El griego Valasopoulo, en 1893, según una comunicación presentada al Congreso de Higiene Mediterránea de 1932, concluyó que las ratas trasmitían el tifus bilioso a los seres humanos (Durich, 1953, p. 203). En 1914, la afección fue reconocida como de causa bacteriana e identificada la espiroqueta como leptospira -nombre adjudicado por Noguchi- por un grupo japonés dirigido por Ryokichi Inada (18741950), que determinó su letalidad para el cobaya. Muy poco después, y de manera independiente, se identificó igualmente en Europa, en los frentes de la Gran Guerra. En los seres humanos esta enfermedad se presenta asociada a condiciones de vida o trabajo que transcurren en contacto estrecho con los animales o sus productos de excreción, pues las leptospiras se eliminan abundantemente por la orina, por lo que precozmente se reconoció su prevalencia en trabajos relacionados con manejo de ganado, cultivos en terrenos húmedos y estancias prolongadas en lugares encharcados como minas, alcantarillas o trincheras (Pumarola, 1984).

En España, la enfermedad de Weil fue descrita clínicamente por un médico ejerciente en Tortosa, Manuel Vila (Vila, 1919) y confirmada bacteriológicamente en el Laboratorio Microbiológico Municipal de Barcelona por Manuel Dalmau (1890-1918). Hallazgos similares se repitieron, de manera esporádica, por España, aunque su cuadro clínico de comienzo brusco, con escalofrío y fiebre alta, y la frecuente presentación bajo formas anictéricas, así como la rápida remisión espontánea de la fiebre, hacía que se confundiera fácilmente con paludismo u otras enfermedades. Por esta razón, se achacó a la tardía desaparición de la malaria autóctona la poca constancia en el registro de leptospirosis (Babudieri, 1953a; Durich, 1953, pp.180181). Pablo Cartañá Castellá (1899-1974) realizó el primer estudio epidemiológico sobre ratas urbanas en Barcelona, mostrando la existencia de infección leptospirósica con ocasión de un brote de peste en 1931, y junto con clínicos como Agustí Pedro i Pons y Juan Surós Forns estudió una pequeña epidemia de espiroquetosis icterohemorrágica por consumo de aguas contaminadas en 1933 (Pedro Pons y Surós, 1933) ${ }^{3}$. A partir de tres casos tratados en el Hospital Provincial, en Valencia se realizó un completo trabajo experimental sobre leptospirosis en la cátedra de Higiene y Microbiología de su Facultad de Medicina (Sanchís Bayarri, et.al., 1935).

Después de la Guerra Civil, los médicos barceloneses seguían estudiando casos esporádicos procedentes del campo que se presentaban todos los veranos en las clínicas universitarias (Profs. Soriano, Pedro Pons, Gibert Queraltó y Gironés) y en las del Hospital municipal (Dres. Trías Fargas, Soler Dopf e Isamat). Los enfermos llegaban de la región del Prat de Llobregat y de Pals y Torroella de Mongrí, sobre todo (Covaleda y Pumarola, 1950). De este modo, el capítulo dedicado a las leptospirosis en el volumen correspondiente a infecciosas del Tratado de Patología y Clínica Médicas dirigido por Pedro Pons (Pedro Pons et al., 1950) mostraba una abundante casuística propia para acompañar un texto de plena actualidad, dotado de la exhaustividad propia del estilo del maestro. Justo Covaleda Ortega (1908-1964), recién llegado a la Facultad de Medicina de Barcelona en 1942 como catedrático de Higiene, Sanidad, Microbiología y Parasitología, apoyó el programa clínico ofreciendo la modernización del diagnóstico de laboratorio. Para ello contó con Agustí Pumarola i Busquets (1920-1987), a quien asignó como tema de tesis el estudio de la Leptospira icterohaemorrhagiae, sus medios de cultivo e inoculación experimental (Pumarola, 1947). Una ilustración de laboratorio correspondiente a esta tesis ilustraba el capítulo del Tratado de Pedro Pons citado. La presentación de la capacidad del laboratorio barcelonés para efectuar eficazmente el diagnóstico de leptospirosis, vehiculada por la revista del Consejo General de Colegios Médicos (Covaleda y Pumarola, 1950), lo convirtió en el centro español de referencia.

Al igual que en Cataluña, en la provincia valenciana tampoco habían dejado de presentarse casos anualmente en la época de la recogida del arroz, con particular intensidad en 1942, 1949 y 1951 (Sanz Sols, 1952). Al no ser una enfermedad de declaración obligatoria no fue recogida por las estadísticas oficiales. Sin embargo, una vez superadas las urgencias de la postguerra, su Jefe Provincial de Sanidad, Durich, quien tenía en su currículo la identificación de un caso de fiebre icterohemorrágica en 1924, prestó atención a los avisos que le llegaron de la provincia y en 1951 llevó ante la Reunión Nacional de Sanitarios Españoles un informe que ponía de manifiesto la existencia de un foco endémico de leptospirosis en los arrozales levantinos y también en los tarraconenses, donde se habían señalado nuevos casos en 1951, alguno producido por otra variedad de Leptospira (Covaleda, Cantarell et al., 1953; Covaleda, Pumarola et al., 1953). El foco estaría mantenido por la enzootia murina y acumulaba sus efectos estacionalmente en los momentos de la siega del arroz. Para confirmarlo bacteriológicamente había recurrido a Covaleda, quien encontró aglutinaciones positivas de una de sus cepas de L. icterohemorrhagiae con el suero de algunos convalecientes (Durich, 1953, pp. 206-207). 
Estos trabajos sirvieron para configurar la leptospirosis como una enfermedad ligada a determinados trabajos que ponían en relación cercana a las personas con los animales portadores: las ratas en el campo, en especial en los arrozales, pero no sólo (Mestre Medina y Santamaría Carmona, 1952), o los cerdos (el primer caso observado en España de meningitis por Leptospira pomona en un cuidador de cerdos fue publicado por Pedro Pons, Farreras y Pumarola, según Covaleda y Pumarola, 1953a). A la vez, la investigación entre animales conocidos como portadores según la bibliografía (ratas del alcantarillado urbano, perros callejeros, cerdos) identificaba la existencia de enzoonosis y llevaba a postular la existencia en nuestro país de infecciones humanas específicas todavía no reconocidas (apuntadas por Pedro Pons y Surós, 1933; Pumarola y Gállego, 1950; Covaleda y Pumarola, 1953b y 1954). A lo largo de las décadas de 1950 y 1960 se continuaron observando y dando a conocer brotes provinciales, junto con la aplicación de medidas de protección que incluyeron la vacunación especifica. En 1969 se determinó la inclusión de la leptospirosis como enfermedad profesional y en 1982 se la incluyó entre las de declaración obligatoria. En la década siguiente, finales del periodo franquista, Pumarola apuntaba que se asociaba a actividades recreativas, por lo que parecía haberse convertido en una enfermedad de las vacaciones (Pumarola, 1975, p. 609).

\section{ESPAÑA EN EL CIRCUITO INTERNACIONAL DE SA- BERES SOBRE LEPTOSPIROSIS}

Como corresponde al momento científico del periodo de entreguerras, las conexiones internacionales resultaban fundamentales para el desarrollo de los países, también en el aspecto sanitario. En esta línea fue decisiva la existencia de la Junta de Ampliación de Estudios e Investigaciones Científicas (JAE), en particular en el fomento de la investigación básica en las universidades, mientras que en el terreno aplicado lo fue mas la actividad de la Comisión de Investigaciones Sanitarias republicana (CIS) y la voluntad explícita de científicos como Gustavo Pittaluga (1876-1956), de quien hay que recordar, junto con su altura intelectual y su activo currículo como gestor de organizaciones sanitarias y periodismo científico, su papel de hombrepuente con la Junta de Sanidad Internacional (IHB) de la Fundación Rockefeller y la Organización de Higiene de la Sociedad de Naciones (Rodríguez-Ocaña, 2010 y 2013; Rodríguez-Ocaña y Borowy, 2008).

No puede entenderse la participación de Manuel Dalmau i Matas (1890-1918), discípulo predilecto de Ramón Turró con quien contactó tras haber sido alumno interno de August Pi Sunyer, sin la pensión de la JAE que le permitió obtener una formación de primer nivel en Halle, con Emil Abderhalden; interrumpida por la guerra, completó la estancia en Harvard, en el laboratorio de Walter Cannon, entre 1916 y 1917. Dalmau pertenecía a la Societat de Biología y era director de la sección de química biológica del Laboratorio Municipal de Barcelona cuando murió víctima de la pandemia gripal en 1918 (Cervera, 1926, pp. 536-537; Galeria, 2016). Demostró la existencia de espiroquetas patógenas para el cobaya en ratas barcelonesas, las cuales presentaban inmunidad cruzada con la cepa Verdun que había obtenido del Instituto Pasteur de París (Dalmau, 1918; Dalmau y Balta, 1919). Louis Martin y André Pettit diseñaron en este centro la prueba de aglutinación de la que procede el test diagnóstico por excelencia, que todavía hoy, modificado, continúa en uso. Los resultados de los trabajos en marcha del malogrado Dalmau, quien correspondió con Pettit, se leyeron en la reunión de abril de 1919 celebrada por la Sociedad Francesa de Biología en la ciudad de Barcelona. No cabe duda de que su muerte prematura supuso un retraso en el devenir de la investigación española sobre leptospirosis.

Entre las aportaciones de la escuela de Pittaluga, encabezada por Sadí de Buen Lozano (1893-1936), recordemos que estuvo la elucidación del complejo etiopatogénico de otra espiroquetosis, la fiebre recurrente española o hispano-africana, con lo que se reforzaron contactos permanentes con los parasitólogos y microbiólogos franceses de las colonias del Norte de África, ya asegurados por la colaboración antipalúdica. Una prueba palmaria fue la celebración del Primer Congreso de Higiene Mediterránea en Marsella en 1932 (Broquet, ed., 1933-1934), al que asistieron, junto con De Buen, otros sanitarios que continuaron activos después de la Guerra Civil, como Juan Durich Espuñes, que sería Jefe Provincial de Sanidad de Valencia, o Pablo Cartañá, futuro director del Instituto Municipal de Higiene de Barcelona. Cartañá tenía experiencia internacional en centros ingleses, como el Laboratorio Lister y el Instituto Wellcome y era malariólogo titulado por la escuela de Brumpt en Francia, becado por la CIS, además de pertenecer a la primera promoción de alumnos de la Escuela Nacional de Sanidad. Otros de los implicados en la primera época de trabajo sobre leptospirosis, como Vicente Sanchís Bayarri y Justo Covaleda, habían estado igualmente becados en el Pasteur de París. Sanchís empleó el suero terapéutico producido por Pettit para determinar la identidad de la espiroqueta hallada en Valencia con la de Dalmau y la francesa. 
Para el momento en que comenzó la Guerra Civil en España, el empleo de las pruebas serológicas para el diagnóstico de leptospirosis había empezado a mostrar la multiplicidad de agentes causales y de sus reservorios animales, al mismo tiempo que la amplitud de variantes antigénicas. Como quiera que se partía de que se trataba de una enfermedad del terreno, asombró el reconocer la identidad antigénica de cepas orientales y europeas. Se inició así un camino que demostró la multiplicidad de la entidad causal, de manera que la entidad nosológica pasó a entenderse como "un conjunto de enfermedades producidas por diversos tipos serológicos de espiroquetas pertenecientes al género Leptospira" (Covaleda y Pumarola, 1953a, p. 19). Para intentar poner orden en aquella selva nosotáxica, se comenzaron a crear Centros de referencia, donde se depositaban muestras de todas las cepas halladas, para permitir el reconocimiento de las que se iban descubriendo en cada país; tal fue el caso del Laboratorio del Instituto Superior de Sanidad de Roma encargado a Brenno Babudieri (1907-1973) en 1936. En 1967, la OMS incluía bajo el nombre Leptospirosis mas de 130 serotipos y 18 serogrupos (Babudieri, 1980); en 2013 se contaban 300 serovares agrupados en 25 serogrupos, sin que exista correspondencia entre las clasificaciones moleculares y serológicas (Junta de Andalucía, 2013).

En la realización de su tesis de doctorado, Pumarola, auxiliar en la cátedra de Covaleda y con un puesto en la sección de Hemoparasitología del Instituto Nacional de Parasitología del CSIC, entró en relación con diversos laboratorios y centros europeos, relación que consolidó con estancias en Lisboa (1947), París (1948) y Roma (1949) y de la que se sirvió para obtener muestras de las distintas variedades serológicas encontradas. Como ejemplo, en un trabajo de dicho autor publicado en 1950 explica que había empleado cepas recibidas de París y de Copenhague, incluyendo especies de Leptospiras todavía no encontradas en España (Pumarola y Gállego, 1950). Como ya hemos advertido, la difusión de la capacidad del laboratorio barcelonés para conseguir un diagnóstico eficaz (Covaleda y Pumarola, 1950) lo convirtió en el centro español de referencia.

Con la entrada de España en la OMS se reforzó esta relación con el entorno europeo, lo que sirvió para reanimar programas de trabajo iniciados con anterioridad a la Guerra Civil. En efecto, establecida dicha relación oficial en 1951 y firmado el acuerdo base para asistencia técnica en enero de $1952^{4}$, el primer acto de colaboración con España se estableció en torno a las enfermedades endemo-epidémicas (Ballester Añón, 2016), mediante un acuerdo específico que entre 1952 y 1956 incluyó leptospirosis y enfermedades venéreas (programa E1) y entre 1955 y 1964 estas últimas y brucelosis (E1.2).

En 1952 se celebró un coloquio europeo sobre zoonosis en Viena, incluyendo sesiones sobre brucelosis, leptospirosis, fiebre $\mathrm{Q}$, rabia y tuberculosis bovina, al que asistieron delegados españoles cuya identidad no hemos podido establecer aún. En la primavera de 1953 un experto italiano comisionado por la OMS e invitado por el Gobierno español, vino a estudiar el problema en España y regresó todos los años siguientes hasta 1956, dentro del mencionado programa de cooperación. Se trataba del ya nombrado Brenno Babudieri, especialista en el estudio biológico de "la leptospirosis de los arrozales" (Babudieri, 1953b), gestor del centro italiano de referencia desde 1936 y productor de la vacuna contra el tifus que había surtido a las tropas italianas del frente oriental europeo durante la IIGM.

1953 fue también el año en que, a finales de septiembre, se reunió en Barcelona, por cuarta vez, el Congreso Internacional de Higiene y Medicina Mediterránea, presidido por Pedro-Pons, donde se habló de leptospirosis dentro de la sección de Higiene, con aportaciones de Covaleda y Pumarola, Babudieri y la francesa Kolochine Erber. La multiplicación de la variedad inherente al género Leptospira consecutiva al establecimiento de líneas permanentes de investigación en los diferentes países había convertido la enfermedad en un gigantesco puzzle nosotáxico y antigénico, lo que exigía el inventario comparado de todas sus manifestaciones locales. De esta forma se situaba España en el mapa occidental, con independencia de su modelo político. Y esa exigencia de la investigación facilitó la relación de los sanitarios españoles con Europa.

Babudieri, que visitó Barcelona, Castellón, Valencia y Sevilla, se presentó con un plan para abordar de forma global el problema a través de una encuesta nacional empleando el diagnóstico de laboratorio estándar, esto es la reacción de aglutinación-lisis frente a las cepas internacionales y regionales. Habría que inventariar las especies predominantes en cada comarca, para lo que exigió el apoyo "de los elementos interesados". Con el respaldo del convenio con la OMS, inició en junio de 1954 la encuesta mediante suministro de 14 cepas de leptospiras en las correspondientes Jefaturas Provinciales de Sanidad (Rey Vila, 1959). Se prestó a recibir en Roma muestras problemáticas en- 
viadas desde España para comparar la receptividad de los sueros de afectados con las cepas controladas en Italia. En el caso castellonense, sirvió de acicate para la formación de un grupo de trabajo específico que se mantendría activo durante una década en torno a Vicente Altava Alegre (1908-1993) y su Centro de Estudios Médico-Biológicos. Igualmente Babudieri generó vínculos con la cátedra de Microbiología de Valencia, pues la tesis de Vicente Sanchís-Bayarri Vaillant, hijo del catedrático autor del estudio experimental publicado en 1935 ya mencionado, y realizada entre 1956 y 1958, utilizó cepas procedentes del laboratorio romano, a su vez obtenidas originalmente de Castellón, Sevilla y Valencia, y del laboratorio de Barcelona (Sanchís-Bayarri Vaillant, 1959).

El programa de colaboración con la OMS sirvió al consultor italiano, ya entonces una autoridad mundial, prestigio que no dejó de incrementar con sucesivas aportaciones científicas de primer orden sobre la biología de estas bacterias, para ensayar en España una vacuna, antes que en su país de origen. Resulta llamativo que, en 1969, ante un auditorio de especialistas, se defendiera de una posible acusación de mala praxis vacunal sobre la prioridad otorgada a España en relación con las pruebas de campo de su vacuna al estilo de la que se acusaba a la URSS en relación con ciertas vacunas contra la gripe ensayadas en Polonia (Babudieri, 1969). Babudieri adujo que el número de especies infectantes en España era muy inferior al de las comunes en Italia, por lo que la vacuna era mas simple. Sólo después de comprobada su inocuidad y efectividad aplicó el modelo a su patria. Por razón de la discrepancia entre las especies causales en Italia y España, por las mismas fechas Pumarola propuso otra hecha a partir de leptospiras obtenidas de enfermos del delta del Ebro, que ensayó in situ en 1953 (Martín Rueda et al., 1955).

\section{LEPTOSPIROSIS COMO DIANA DE UNA INTERVEN- CIÓN SANITARIA RURAL}

El estímulo fundamental para el estudio de la leptospirosis en España vino de la mano de los contactos internacionales, como hemos visto, mientras que la urgencia por actuar se basó en argumentos económicos.

El de las pérdidas económicas consecutivas al padecimiento de las enfermedades endémicas se convirtió en un argumento discursivo recurrente en mítines, conferencias y escritos periodísticos en el siglo XX, en especial en los momentos de agitación sanitaria como los vividos en torno a 1920 (Rodríguez-Ocaña, 2007). En los últimos momentos de vigencia de la malaria autóctona en España, José Fernández Maruto, último Director del Instituto Nacional Antipalúdico, calculó los costes de la endemia en casi 9.000 millones de pesetas, en pesetas de 1959, frente a apenas 38 millones empleadas en los programas de intervención desde 1920. Y ello sin añadir otras ventajas económicas indirectas que habría que asignar a la erradicación del endemismo palúdico tales como el desarrollo agrícola por aumento de las posibilidades de explotación en nuevas tierras, el fomento de la obra pública y el crecimiento del turismo (Fernández Maruto, 1964, p. 94).

Por su parte, la anquilostomiasis que fue el objeto inicial de la colaboración con la International Health Board de la Fundación Rockefeller se vinculó expresamente al mundo minero, donde primero se había detectado en España y desde donde se construyó como auténtico «peligro de hecatombe». La infección propiamente rural se comenzó a detectar de manera puntual a partir de bien entrada la década de 1920, y dio lugar a intervenciones en zonas de gran riqueza agrícola como la Huerta de Murcia, la Ribera Baja del Júcar, la Plana de Castellón y los arrozales del delta del Ebro (Rodríguez-Ocaña y Menéndez-Navarro, 2009). La enfermedad individual se transformaba en social por sus efectos sobre la productividad y riqueza agrícolas al constatar que la anquilostomiasis generaba un «serio problema que afecta a la economía de la vega del Segura» (Guillamón, 1927, p. 3).

Coyunturalmente el contacto con la OMS coincidió en 1952 con un brote epidémico que presentó caracteres de gravedad y que fue leído como una amenaza económica, "daño irreparable a la economía nacional" según los autores que estudiaron la leptospirosis sufrida en los arrozales de Castellón (Altava et al., 1953). En efecto, el estudio calculaba ese coste a partir del número de casos que habían controlado en una pequeña zona de la provincia, mediante extrapolación al conjunto de la provincia y a la totalidad de la España arrocera, y que supondrían mas de diez millones de pesetas a nivel estatal.

Se definió la leptospirosis de los arrozales como el principal foco endémico en España (Covaleda y Pumarola, 1953a). Las altas tasas de incidencia en los trabajadores, concentradas en el momento de la siega, y el largo plazo de recuperación exigido por la enfermedad creaban un "importante problema sanitario, social y económico". Citaban cifras de incidencia de entre el 6 y el $50 \%$ entre los componentes de las cuadrillas de segadores de arroz, según un informe valenciano de 1951, y subrayaban su comprobación empírica de la alta prevalencia de casos anictéricos, 
de difícil diagnóstico clínico, mientras que la profunda astenia residual era responsable de la inhabilitación para el trabajo de los afectados pese a la levedad de la enfermedad (Covaleda, Cantarell et al., 1953).

Otro argumento, apuntado por Altava et al. (1953) y sustentado con mayor convicción por Juan Durich, en un trabajo sobre los distintos brotes de posguerra (Durich, 1953), centraba el problema coyuntural en el peligro para la continuidad de las tareas de siega del arroz. La siega era el momento crucial del ciclo del cultivo y era el momento en que aparecía la leptospirosis de manera epidémica. Si bien la letalidad de esta afección era muy baja, los trabajadores temían el padecimiento, porque los obligaba a abandonar el trabajo en el momento mejor pagado y amenazaba con dejarlos sin beneficio por el coste del tratamiento antibiótico, pues lo ganado se les iba en pagar medicinas. Recordemos que la principal baza de la lucha antipalúdica radicaba en que la labor de despistaje y tratamiento era totalmente gratuita. Altava y Durich planteaban el riesgo de la ausencia de mano de obra suficiente, con la consiguiente ruina de los cultivadores, por lo que sugirieron el reconocimiento de la leptospirosis del arrozal como enfermedad profesional, como lo era en Italia, lo que se concedió, tardíamente, en 1969. Por eso, la Cooperativa Nacional Arrocera se comprometió a abonar todos los gastos derivados del tratamiento de los afectados valencianos en 1952, algo que no mantuvo en los años siguientes aunque sí la colaboración en materia de prevención mediante actuaciones sobre el terreno.

La Escuela Nacional de Sanidad intervino en 1954 para encargar a la Jefatura Provincial de Sanidad (JPS) de Valencia el estudio de la endemia en su provincia así como el ensayo de métodos profilácticos de cara a su posterior extensión, para lo que dicha Jefatura conectó con el grupo del Instituto Nacional de Parasitología y aplicó la vacuna allí confeccionada. En el delta del Ebro y en Valencia se mantuvo una campaña anual de vacunación que se aplicó a unos cientos de trabajadores (Durich y Pumarola, 1955), mientras que en la provincia de Castellón se introdujo la vacuna hecha por Babudieri (Altava et al., 1955). Según resume Pumarola en su manual de Medicina Preventiva de 1975, la vacuna se mostró eficaz, pues en un momento en que se detuvo su aplicación anual (1963) se registraron más de 300 casos (Pumarola, 1975, pp. 611-612).

No fue hasta 1964 cuando se acometió la única encuesta serológica nacional en la totalidad de las 10 provincias arroceras, las tradicionales y las recién incorporadas, donde también se habían advertido casos, como en Gerona (Covaleda, Pumarola et al., 1955) o en Sevilla (Rey Vila, 1959; Rey Vila, et al., 1959). Se recogieron un total de 945 muestras, con el resultado de $42 \%$ de sueros positivos (al $1 / 10$ ), o un $30,3 \%$ (al $1 / 50$ ), con lo que se descontaban posibles reacciones inespecíficas. Los sueros sugerentes de positividad reciente sumaban el $10 \%$, lo que equivalía al número de trabajadores infectados en la última siega y eran positivos a cinco serotipos (L. icterohemorrhagiae, $72 \%$, L. ballum, 19,8\%, L. grippotyphosa, 5,2\%, L. pomona, 1\%, L. sejro, 2,1\%). La distribución de positivos por provincias mostraba tres grupos, el de las zonas clásicas (Valencia, Gerona, Tarragona y Alicante) con alrededor del $15 \%$ de población trabajadora infectada, el grupo novel fuerte (Sevilla) con el doble de porcentaje y el grupo novel débil (Cáceres, Albacete, Huesca) con mínima prevalencia de entre 1 y $3 \%$ (Gimeno de Sande y Pumarola, 1967). La provincia de Castellón, una zona clásica, mostraba resultados entre los mas bajos, lo que indicaba que en ella se había realizado el control sistemático de esta infección. La conclusión de la encuesta, que sirvió para montar una comunicación al congreso de Microbiología de Moscú (1966), era que hacía falta reforzar las campañas de vacunación. En 1964 se vacunaron 2.064 segadores y en 1965, 3.012 (Gimeno de Sande y Pumarola, 1967, p. 131) en las zonas levantinas -únicas que se identifican- y, según Pumarola (1975), en algún momento que no especifica se consiguió la desaparición de la enfermedad de las zonas endémicas tradicionales.

La complejidad antigénica del género parasitario hacía muy complicada la confección de la vacuna, por lo que se emplearon también métodos físicos (cementado de canales) y toxicológicos (raticidas) para prevenir la enfermedad (Durich, 1953; Altava y Barrera, 1961). En todo caso, las iniciativas para combatir la enfermedad fueron provinciales, sujetas a distintos grados de colaboración con el Sindicato de empresarios arroceros y no vinculadas a ninguna reglamentación común. Cada JPS tenía la autonomía para plantear el tipo de intervención a seguir. Lo mismo parece ocurrir con las vacunaciones, en un momento en que las energías de la D.G. de Sanidad parecían concentradas alrededor de la poliomielitis.

Otra derivada tardía de la atención a la leptospirosis de los arrozales fue el frustrado intento de conseguir una atención específica a la medicina del trabajo rural mediante la creación de Centros de Medicina Social Agraria, en línea con lo que se hacía en Francia o en Polonia y se vehiculaba como preocupación interna- 
cional en los Congresos de Medicina Agrícola, de lo que he tenido ocasión de hablar en una publicación anterior (Rodríguez-Ocaña, 2012).

\section{AUSENCIA DE RELACIÓN CON LA POBLACION}

Un componente fundamental en la acción sanitaria en sentido médico-social es el ejercicio de una labor educativa de los saberes médicos e higiénicos correspondientes entre la población diana de aquélla (Rodríguez-Ocaña y Molero, 1993). Con ello no solo se esperaba una modificación en los comportamientos de riesgo señalados, sino una mayor cercanía con los profesionales expertos y mayor confianza en su actuación.

El ejemplo de la intervención contra la anquilostomiasis en la Huerta de Murcia resulta significativo (Guillamón, 1927). Se inició en 1927 con un proyecto informativo que abarcó 29 escuelas y unos 4.000 asistentes y consistía en conferencias, exposición de carteles (sobre normas higiénicas, evolución del parásito y anomalías de la sangre), reparto de un millar de cartillas y de 7.000 juegos de cinco láminas que reproducían los carteles e incluían al dorso un resumen de lo explicado. La lucha antipalúdica, por su parte, contó también con un importante despliegue de medios de propaganda sanitaria, desde antes incluso de su inicio organizado, como el Cartel informativo sobre la prevención del paludismo, realizado por José Verdes Montenegro, y aceptado por el Ministerio de Instrucción Pública para su compra por las Escuelas (R.O. 25 de mayo de 1905, Gaceta del 3 de junio), hasta los folletos explicativos sobre la naturaleza de la enfermedad y el modo de prevenirse, de uno de los cuales se repartieron 50.000 ejemplares en 1943 (Buen, 1922; Dirección General de Sanidad, s.a.). Hasta tal punto se tenía en consideración la actividad educativa que el Ministro de la Gobernación apuntó que una de las causas de la gran propagación del mal en la posguerra inmediata era la falta de propaganda, por la incultura existente entre las poblaciones aquejadas (Rodríguez-Ocaña y Perdiguero, 2009).

Esta queja recogía una preocupación continua entre los sanitarios españoles, como se puede encontrar en otros contextos, desde la culpabilización a las madres por su ignorancia en el cuidado de los lactantes (Rodríguez-Ocaña y Perdiguero, 2006) a la acusación de dejadez o desidia imperdonable frente al tracoma enunciada en 1927 y repetida en 1941 (BernabeuMestre y Galiana-Sánchez, 2012, p. 101). Pero existieron modos y maneras distintas de expresar y contextualizar dicha preocupación que se han estudiado en el contexto de la anquilostomiasis comparando los discursos epidemiológicos de antes y después de la Guerra (Rodríguez-Ocaña y Menéndez-Navarro, 2009). La diferencia estriba en la profundidad del desprecio que encierra el juicio médico.

En el caso de la leptospirosis no he hallado pruebas de ninguna actividad propagandística o educativa, salvo la de la "propaganda por los hechos" consecutiva a la eficacia de las intervenciones preventivas. Haría falta una exploración en el terreno de la actuación de las Hermandades de Labradores y Ganaderos para confirmarlo. En lo que se refiere al discurso de los epidemiólogos, a diferencia de los análisis sobre el brote de uncinariasis de la provincia de Madrid, este se centró en las condiciones de trabajo en el arrozal para los casos del mundo rural y en las condiciones de vida miserables para los casos urbanos, como vivir de la recogida de objetos perdidos en las aguas de las alcantarillas por parte de los habitantes de la barriada barcelonesa de Somorrostro, por ejemplo (Covaleda y Pumarola, 1952), sin referencia ninguna a supuestas lacras culturales o morales.

La justificación económica y productivista sirvió de motor a la intervención sanitaria en todos los casos. Ahora bien, de acuerdo con los planteamientos del reformismo en ciertos momentos dicha justificación se acompañó de la voluntad de inclusión de una población tradicionalmente excluida. Como expresó con absoluta claridad Pittaluga, en correspondencia con su pertenencia a la Liga Española de los Derechos Humanos (Pittaluga, 1927, p. 32):

\footnotetext{
"No espero ni la desaparición de los mosquitos ni la extinción de los parásitos; solo deseo ser capaz de procurar ese mínimo de bienestar físico sin el que es imposible tener dignidad humana ni conciencia de ciudadano".
}

Manifestaciones explícitas de este tipo desaparecieron con el fin de la guerra, de modo que en la primera época del franquismo las propuestas estratégicas sanitarias que se hicieron se vincularon con el bienestar y el poderío de la Patria, dentro de los objetivos imperiales del Nuevo Estado y sin mención alguna al bienestar ciudadano; sin embargo, en alguna medida permanecieron como parte del ethos profesional salubrista. En enero de 1952 no tuvo empacho el Gobierno en firmar un acuerdo de asistencia técnica con la OMS que estaba enfocado genéricamente hacia "to promote the economic and social progress and development of peoples" 5 . Otra cosa es que dicho objetivo presidiera la actividad sanitaria, mucho mas preocupada, bajo el franquismo, del escaparate que de los contenidos reales. 


\section{CONCLUSIÓN}

La evolución de la Medicina Social en España se sustentó sobre dos patas: la incorporación de nuestros expertos al sistema mundial del conocimiento y la justificación económica y productivista como motor de la intervención sanitaria. Su presencia en el mundo urbano se redujo antes debido a la entrada en vigor del Seguro Obligatorio de Enfermedad, mientras que se mantuvo por más tiempo en las campañas de intervención rural, incluyendo la colaboración directa y la financiación de organismos internacionales como la Fundación Rockefeller y la OMS. En los respectivos convenios se mezclaron las conveniencias políticas a nivel de estado -como conseguir una legitimación internacional en situaciones de dictadura- y las conveniencias curriculares para los actores implicados, que buscaron apuntalar sus trayectorias profesionales dentro de España con el contacto permanente con centros punteros a nivel internacional a través del cual asegurar su promoción y la de sus discípulos directos. Resulta significativo que las medidas innovadoras de tipo científico $u$ organizativo en relación con la leptospirosis durante el franquismo -ninguna en sentido educativo- se tomaran en la vecindad de eventos internacionales (Conferencia de Barcelona, 1953; Congreso de Microbiología de Moscú, 1966) en los que podían trasmitir una imagen dinámica e innovadora de la Sanidad española. Lo que coincidía, en enfermedades donde los humanos eran vehículos necesarios de la infección como la anquilostomiasis, con una consideración de desprecio a una población subordinada.

La existencia de una presión negativa sobre la productividad laboral fue el estímulo para la intervención pública, como ocurrió en el entorno del arroz, cuyo modo de aparecer y desarrollarse prueba el agotamiento del modelo médico-social anterior a la Guerra Civil, pese a las potencialidades que encerraba, tal y como mas tarde reconocieron consultores internacionales ${ }^{6}$. Así podemos observar la falta de participación de los centros secundarios (comarcales) de Higiene rural, el absoluto protagonismo de los Jefes Provinciales, el errático devenir de las vacunaciones contra la leptospirosis en la década de 1960 y la ausencia de una actividad divulgativa pautada: la lucha contra la leptospirosis se libró básicamente en torno a las propuestas del laboratorio. La participación de la OMS en este campo fortaleció de hecho esta tendencia.

\section{AGRADECIMIENTOS}

Trabajo realizado en el marco del Proyecto HAR201570688-C2-1-P (MINECO-FEDER). Una versión preliminar de este trabajo se presentó al XI Congreso de la ADEH, Cádiz 21 a 24 de junio de 2016.

\section{NOTAS}

1. Los dispensarios especializados se suprimieron en las poblaciones no capitales de provincia por Orden del Ministerio de Trabajo, Previsión y Sanidad de 1 de julio de 1935, Gaceta del 13.

2. Frank W. Reynolds. A Brief Enquiry into the Organization and Functioning of Venereal Disease Control Services in Spain. October 1951. Archivo de la Organización Mundial de la Salud, Ginebra, WHO 2/DC-VD 2-8.

3. La participación de Cartañá en esta epidemia, aunque no figura en el trabajo impreso, es confirmada por (Covaleda y Pumarola, 1953a).

4. Basic agreement concerning technical assistance. WHO2$\mathrm{PH}-2 / 5 / 50$. Archivo de la Organización Mundial de la Salud, Ginebra.

5. Basic agreement ... WHO2-PH-2/5/50. Archivo de la Organización Mundial de la Salud, Ginebra. Se trata de una copia empleada con efectos informativos donde no constan las firmas, por lo que desconocemos la autoridad española responsable.

6. Farrington, C. Fraser. Informe sobre la organización de los servicios sanitarios en España. Misión ejecutada entre el 28 de septiembre y el 17 de diciembre de 1967. Ejemplar mecanografiado, Biblioteca central de la OMS, Ginebra. Este informe, exhumado por la Profesora Rosa Ballester, está siendo en la actualidad objeto de análisis. 


\section{BIBLIOGRAFÍA}

Altava, Vicente, Villalonga, I., Barrera, M. y Marín, C. (1953), "Nuevas ideas sobre la leptospirosis de los arrozales. Epidemiología y profilaxis en los arrozales de Castellón", Rev Clin Esp, 48 (5), pp. 292-300.

Altava, Vicente, Barrera, M., Villalonga, I., Gil, P., Marín, C. y Babudieri, B. (1955), "Vacunación como profilaxis en la leptospirosis de los arrozales de Castellón", Rev San Hig Pub, 29, pp. 167-170.

Altava, Vicente y Barrera, M. (1961), "La desratización como profilaxis de la leptospirosis en los arrozales", Rev San Hig Pub, 35, pp. 647-655.

Babudieri, Brenno (1953a), "Estado actual del problema de las leptospirosis", Med Esp, 29, pp. 323-336.

Babudieri, Brenno (1953b), "La leptospirosi delle risaia italiane". En: Rodríguez López-Neyra, Carlos et al. (coord.), Symposium de Higiene. IV Congreso Internacional de Higiene y Medicina Mediterránea, Barcelona, Barcelona, Talleres Tip. Ariel, pp. 45-51.

Babudieri, Brenno (1969), “L’azione sanitaria internazionales nel campo del tracoma e della leptospirosi", Ann Sanita Pubblica, 30 (6), pp. 911-928.

Babudieri, Brenno (1980), “Leptospira genere”. En: Vella, Luciano (dir.), Enciclopedia medica italiana, vol. 8, 2a ed., Firenze, USES Edizioni Scientifiche; cols. 1420-1423.

Ballester Añón, Rosa (2016), España y la Organización Mundial de la Salud en el contexto de la historia de la salud pública internacional (1948-1975). Discurso de recepción de la académica electa. Valencia, Real Academia de Medicina de la Comunidad Valenciana.

Bernabeu-Mestre, Josep y Galiana-Sánchez, M.E. (2012), "Determinantes laborales y de género en la epidemiología del tracoma en la España contemporánea". En: Cohen, Arón (ed.), El trabajo y sus riesgos en la época contemporánea. Conocimiento, codificación, intervención y gestión, Barcelona, Icaria Editorial y Universitat de Barcelona, pp. 91-122.

Bernabeu-Mestre, Josep, Galiana-Sánchez, M.E. y Cremades Monerris, A. (2013), "Environment and health in a povertyrelated disease: epidemiology of trachoma in contemporary Spain, 1900-1965", História, Ciências, Saúde - Manguinhos; 20 (4), pp. 1065-1619.

Broquet, Charles (ed.) (1933-1934), Hygiène méditerraneénne. ler Congrès international. Marseille, 20-25 septembre 1932, tenu sous les auspices de la Faculté de Médecine de Marseille. Rapports et comptes rendus, 2 vols., Evreux et Paris, impr. Hérissey et libr. J.-B. Baillière et fils.
Buen, Sadí de (1922), El paludismo en el campo, Madrid, Espasa Calpe [catecismos del agricultor y del ganadero]. Reediciones en 1926, 1929 y 1935.

Cervera, Leandro (1926), "Vida y obras de Turró. Notas para una biografía y bibliografía", Rev Hig San Pecuarias, 16 (810), pp. 527-546.

Covaleda Ortega, Justo y Pumarola Busquets, Agustín (1950), "Las leptospirosis europeas y sus métodos de diagnóstico", Boletín Cultural e Informativo del Consejo General de Médicos de España, no50, pp. 21-28.

Covaleda, Justo y Pumarola Busquets, Agustín (1952), "Estudio epidemiológico de la enfermedad de Weil en Cataluña con ocasión de un pequeño brote epidémico", Rev San Hig Pub, 26, pp. $189-198$.

Covaleda, Justo y Pumarola Busquets, Agustín (1953a), "La leptospirosis en España”. En: C. Rodríguez López-Neyra et al. Symposium de Higiene. IV Congreso Internacional de Higiene y Medicina Mediterránea, Barcelona 1953. Barcelona: Talleres Tip. Ariel, pp. 19-33.

Covaleda Ortega, Justo y Pumarola Busquets, Agustín (1953b), "Sobre el grado de infección de los perros de Barcelona por L. Canicola y L. Icterohaemorrhagiae", Rev Iber Parasitol, 13, pp. $185-191$

Covaleda Ortega, Justo y Pumarola Busquets, Agustín (1954), “Aglutinación de leptospiras L. pomona, L. mitis y L. icterohaemorrhagiae por los sueros de cerdos aparentemente sanos", Rev Iber Parasitol, 14 (1), pp. 3-12.

Covaleda, Justo, Cantarell, I. y Pumarola, Agustín (1953), "Brote epidémico de leptospirosis anictérica originado por L. icterohaemorhagiae en los trabajadores de arrozales de la región de Camarles (Tortosa) [1951]", Med. Clin., 20 (2), pp. 75-79.

Covaleda, Justo, Pumarola, Agustín y Cantarell, I. (1953), "Leptospirosis por L. Ballum en los trabajadores de arrozal de la región de Camarles (Delta del Ebro)", Rev Iber Parasitol, 13 (2), pp. 289-298.

Covaleda, Justo, Pumarola, Agustín y Mascaró, J. (1955), "Leptospirosis por Leptospira Grippo-Typhosa en los arrozales de la provincia de Gerona". En: Libro homenaje al prof. Lopéz Neyra de la Revista Ibérica de Parasitología, Granada, Instituto Nacional de Parasitología, pp. 893895.

Dalmau, Manuel (1918). “L’Espiroquetosi ictero-hemorràgia a Espanta", Treballs de la Societat de Biología, Barcelona, 6, pp. 63-64.

Dalmau et Balta (1919), "Sur l'immunité dans la spirochétose ictérohémorragique", CR Soc Biol, 82, pp. 489-490. 
Dirección General de Sanidad, Instrucciones sobre el Paludismo, Madrid, s.i; s.a [1943].

Durich, Juan (1953), "La leptospirosis entre los cultivadores de los arrozales", Rev San Hig Pub, 27, pp. 179-220.

Durich, Juan y Pumarola, Agustín (1955), “La endemia de leptospirosis en la provincia de Valencia. Ensayo de métodos profilácticos (1954)", Med Esp, 34 (197), pp. 83-90.

Fernández Maruto, J. J. (1964), "Trascendencia sanitaria y económico-social de la erradicación del paludismo en España", Rev San Hig Pub, 38, pp. 89-117.

Galeria de metges catalans (2016), "Dalmau i Matas, Manuel", libre acceso en http://www.galeriametges.cat/galeria-fitxa. php?icod=FDF, consultada el 28 de abril de 2016.

Galiana-Sánchez, María Eugenia, Cremades Monerris, A. y Bernabeu-Mestre, J. (2010), "Sanitary campaigns against trachoma in Spain". En: Andresen, Astri; Barona, Josep L.; Cherry, Steve (eds.), Making a New Countryside. Health Policies and Practices in European History ca. 1860-1950. Frankfurt a M: Peter Lang, pp. 101-111.

Gimeno de Sande, Alfredo y Pumarola, Agustín (1967), “La leptospirosis de los arrozales de España (Encuesta epidemiológica nacional 1964-1965)", Rev San Hig Pub, 41, pp. 119-133.

Guillamón, Antonio (1927), “El problema de la anquilostomiasis en la Huerta de Murcia. Memoria de la campaña de divulgación autorizada por el Excmo. Ayuntamiento y de los trabajos realizados". Separata de Estudios Médicos, 45.

Junta de Andalucía y Red Nacional de Vigilancia Epidemiológica (2013), Protocolo de Vigilancia y Alerta de Leptospirosis, Acceso libre en http://www.juntadeandalucia.es/salud/ export/sites/csalud/galerias/documentos/p_4_p_1_vigilancia_de_la_salud/leptospirosis.pdf, consultada el 14 de octubre de 2015

Landouzy (1883), "Typhus hépatique”, Gazette des hôpitaux civils et militaires, 56 , pp. 913-914.

Marset Campos, Pedro, Rodríguez-Ocaña, Esteban y Sáez Gómez, José Miguel (1998), "La Salud Pública en España". En: F. Martínez et al. Salud Pública, Madrid, MacGraw-Hill Interamericana, pp. 25-47

Martín Rueda J., Covaleda Ortega, Justo, Pumarola Busquets, Agustín, Ema Berenguer, J. y Cantarell Fontcuberta, I. (1955), "La vacunación en la profilaxis de la leptospirosis en los arrozales. Prueba de campo en el delta del Ebro. Verano 1953". En: Libro homenaje al prof. López Neyra de la Revista Ibérica de Parasitología, Granada, Instituto Nacional de Parasitología, pp. 123-131.

Martínez Antonio, Francisco Javier y Molero Mesa, Jorge (2002), "Las campañas sanitarias como paradigma de la acción social de la medicina". Trabajo social y salud, 43, pp. 119-148.
Mathieu, Albert (1886), "Typhus hépatique bénin; rechute, guérison". Revue de Medecine (Paris), 6, pp. 633-639.

Mestre Medina, Joaquín y Santamaría Carmona, Germán de (1952), "Sobre una epidemia de leptospirosis gripotifosa en la provincia de Toledo", Boletín Cultural Informativo del Consejo Superior de Colegios Médicos, septiembre, pp. 39-41.

Pedro i Pons, Agustí y Surós y Forns, Josep (1933), "Consideraciones sobre una epidemia de espiroquetosis icterígena”, Revista Medica de Barcelona, 20, pp. 291-306.

Pedro i Pons, Agustí, Farreras Valentí, Pere y Surós Hornos, Josep (1950), Enfermedades infecciosas, intoxicaciones, Enfermedades Profesionales y por Agentes físicos, vol VI del Tratado de Patología y Clínica Médicas, dirigido por A. Pedro i Pons, Barcelona, Salvat Editorial.

Pittaluga, Gustavo (1927), Médicos e ingenieros en la lucha contra el paludismo. Conferencia pronunciada el día 7 de abril de 1927 en la Escuela de Ingenieros de Caminos de Madrid, Madrid.

Pumarola Busquets, Agustín (1947), "Leptospira icterohaemorrhagiae. Aportación al estudio de los medios de cultivo e inoculación experimental", Rev Iber Parasitol, 7 (3), pp. 338-445.

Pumarola Busquets, Agustí (1975), "Leptospirosis". En: Pumarola Busquets, Agustí et al. Medicina Preventiva y Social. Higiene y Sanidad ambiental, vol. 1, Madrid, Amaro Ediciones y Publicaciones, pp. 601-612.

Pumarola Busquets, Agustí (1984), "Leptospira”. En: Pumarola, Agustí et al., Microbiología y Parasitología Médica, Barcelona, Salvat, pp. 518-523.

Pumarola Busquets, Agustín y Gállego Berenguer, J. (1950), "Estudio epidemiológico y microbiológico de la leptospirosis murina en Barcelona". Rev Iber Parasitol; 10, pp. 333-336.

Rey Vila, Fernando (1959), "Leptospirosis en los arrozales de Isla Mayor del Guadalquivir". Libro de Actas de la V Reunión Nacional de Sanitarios, Madrid, abril de 1959, pp. 895-897.

Rey Vila, Fernando, Lázaro Brouet, J. y Díaz Rodríguez, J.A. (1959), "Leptospirosis en los arrozales de Isla Mayor del Guadalquivir (Sevilla)", Rev San Hig Pub, 33, pp. 80-88.

Rodríguez-Ocaña, Esteban (2007), “Medicine as a Social Political Science: The Case of Spain c. 1920", Hygiea Internationalis, 7 (1), pp. 37-52.

Rodríguez-Ocaña, Esteban (2008), "La Sanidad en la II República española, 1931-1939". En: El Centro Secundario de Higiene rural de Talavera de la Reina. 75 aniversario de una experiencia modernizadora en la Sanidad, Talavera de la Reina, Colectivo de Investigación Histórica Arrabal, pp. 5-12.

Rodríguez-Ocaña, Esteban (2010), “La JAE y la consolidación de la Salud Pública en España". En: Sánchez Ron, 
José Manuel y García-Velasco, José (eds.), 100 JAE. La Junta para Ampliación de Estudios e Investigaciones Científicas en su centenario, Madrid, Publ Residencia de Estudiantes, pp. 601-623.

Rodríguez-Ocaña, Esteban (2012), “Trabajo campesino, enfermedad y prevención en la España del Siglo XX". En: Cohen, Arón (ed.), El trabajo y sus riesgos en la época contemporánea. Conocimiento, codificación, intervención y gestión, Barcelona, Icaria Editorial y Universitat de Barcelona, pp. 29-56.

Rodríguez-Ocaña, Esteban (2013), “Pittaluga Fattorini, Gustavo". En: Diccionario Biográfico Español, vol. XLI: De "Pérez Cecilia" a "Porcell", Madrid, Academia de la Historia.

Rodríguez-Ocaña, Esteban, Ballester Añón, Rosa, Perdiguero, Enrique, Medina Doménech, Rosa Mạ. y Molero, Jorge (2003), La acción médico-social contra el paludismo en la España metropolitana y colonial del siglo XX, Madrid, CSIC.

Rodríguez-Ocaña, Esteban y Borowy, Iris (2008), “Gustavo Pittaluga (1876 - 1956) Science as a Weapon for Social Reform in a Time of Crisis". En Borowy, Iris, Hardy, Anne (eds.), Of Medicine and Men. Biographies and Ideas in European Social Medicine between the World Wars, Frankfurt a.M.: Peter Lang, pp. 173-196.

Rodríguez-Ocaña, Esteban y Martínez Navarro, Ferrán (2008), Salud pública en España: de la Edad Media al siglo XXI, Granada, Escuela Andaluza de Salud Pública.

Rodríguez-Ocaña, Esteban y Menéndez-Navarro, Alfredo (2006), "Higiene contra la anemia de los mineros. La lucha contra la anquilostomiasis en España (1897-1936)", Asclepio, 58 (1), pp. 219-248.

Rodríguez-Ocaña, Esteban y Menéndez-Navarro, Alfredo (2009), "Hookworm in rural Spain, 1910-1960: shifting para- digms around the Civil War", J Epidemiol Community Health, 63 (8), pp. 670-674.

Rodríguez-Ocaña, Esteban y Molero Mesa, Jorge (1993), "La cruzada por la salud. Las campañas sanitarias del primer tercio del siglo veinte en la construcción de la cultura de la salud". En: Montiel, Luis (coord.) La Salud en el Estado de Bienestar. Análisis histórico, Madrid, Editorial Complutense, pp. 133-148.

Rodríguez-Ocaña, Esteban y Perdiguero, Enrique (2006), "Ciencia y persuasión social en la medicalización de la infancia en España, siglos XIX y XX", História-Ciências-Saúde - Manguinhos, 13, pp. 303-324.

Rodríguez-Ocaña, Esteban y Perdiguero Gil, Enrique (2009), "Las campañas antipalúdicas en España". En: Malaria: exposición celebrada en la Biblioteca Nacional del 17 de marzo al 7 de junio de 2009 [comisarios, José A. Nájera y Antonio González Bueno], Madrid, Biblioteca Nacional, pp. 97-112.

Sanchís Bayarri, Vicente, Montoliu Volant, C. y Sabina Parra, F. (1935), "Contribución al estudio de la espiroquetosis icterohemorrágica", Rev San Hig Pub, 10 (2), pp. 113-124.

Sanchís Bayarri Vaillant, Vicente (1959), "Contribución al estudio de los Leptospiras de las aguas". Med Esp, pp. 432-447.

Sanz Sols, Rafael (1952), "Leptospirosis en la provincia de Valencia", Medicamenta, 216, pp. 269-273.

Vila i Olesa, Manuel (1919). "La enfermedad de Weil en el Delta del Ebro”. Siglo médico, 1919, pp. 110-132.

Weil, Adolph (1886), “Ueber eine eigenthümliche, mit Milztumor, Icterus and Nephritis einhergehende, acute Infectionskrankheit", Dtsch Arch klin Med, 39, pp. 209-232. 